\title{
How we started CSCW
}

\author{
Computer-supported cooperative work (CSCW) seeks to understand and refine the impact of technology on \\ how groups work together. Irene Greif recounts how the field began in 1984 at a meeting stimulated by trends in \\ distributed systems, networks and office automation.
}

\section{Irene Greif}

\begin{abstract}
$\mathrm{n}$ the 1980s, processes that used to be handled person to person were being automated, shifting control of technology from centralized IT departments to personal computer users. Such technology promised productivity gains but was actually wreaking havoc. Meanwhile, anthropologists of the
\end{abstract} day began studying offices, as they had previously studied indigenous cultures, coming to understand the impact of computer technology on informal processes that were critical to the operation of an office. Having worked in the distributed systems group at MIT's computer science lab before moving to their office automation group, I was lucky to be at the centre of both system-oriented and people-oriented perspectives on these issues.

Sensing these trends, Paul Cashman of Digital Equipment Corporation and I decided to run a workshop in 1984 with a diverse group of people - computer scientists in fields from artificial intelligence to distributed systems, sociologists, futurists and business management researchers - that we felt were working on similar problems. We coined the phrase 'computersupported cooperative work' and invited about 25 people to attend. All invitees accepted and sent us papers to be shared in advance. We were lucky that few of them opened the collection before starting on their way to the meeting - puzzlement was the initial reaction of many, wondering what do these projects have to do with each other? By the end of the workshop, though, the question had been answered.

We agreed there was indeed an area of overlap that merited a forum and a designation. And the emphasis would be different from the young field of computerhuman interaction, which focused on individual interactions with computers, rather than issues of groups or organizations.

In 1986, while home on maternity leave, I was invited to co-chair a conference on team software development, sponsored by the Microelectronics and Computer Technology Corporation in Austin, Texas (Fig. 1). I suggested that we broaden the scope of the conference to computer-supported cooperative work more generally. Co-chair Herb Krasner agreed, and, with the shortened acronym CSCW, we were off.

That conference revealed a disconnect that set our agenda for years. Some of the most creative collaborative systems were being built by people at Xerox PARC, but research papers about them reported only anecdotal stories of their usage. This raised questions for social scientists about the impacts the systems might have in real settings, but the technology was not ready for deployment outside the lab. Meanwhile, sociologists from the Rand Corporation had done a systematic study of real-world use in a federal agency with widely distributed employees, documenting impact and social challenges. But the technologies were so simplistic - character-oriented messaging, for example - that the insight gained from these studies was limited. To Xerox PARC's credit, they were also among the first labs to employ anthropologists to study office interactions, but again, only for technologies already in widespread use.

The conflict energized us to focus the field on bridging the gap between advanced technologists and the social scientists best equipped to assess the impact of technology. This disconnect between technical advances and cultural acceptance was reflected in another landmark workshop in 1986 in Palo Alto, California - the ACM Conference on the History of Personal Workstations. At this meeting, Doug Engelbart, inventor of the mouse, spoke about many of his other inventions and showed excerpts from his 1968 demo, which is sometimes referred to as the 'mother of all demos. This demo included remote presenters, shared workspaces and numerous other features of collaboration technology that we seemed to be rediscovering in 1986. He had also already recognized the importance of human psychology in designing, implementing and deploying these systems. He was puzzled that we seemed to just be rediscovering old work, but the lesson I took away was that CSCW represented a growing recognition of the challenges of synchronizing the social and technical, and the need to revisit both over time.

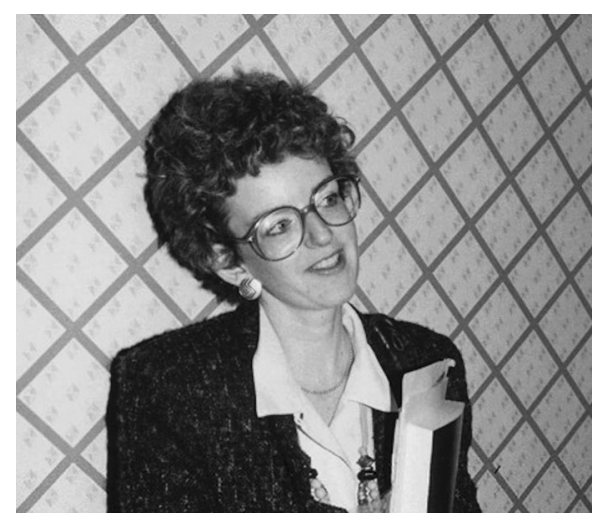

Fig. 1 | Irene Greif at CSCW in 1986. Photo by Ben Shneiderman.

The tension between technological advance and social readiness was key to progress and remains so today. We live with so many of the technologies barely imagined in the 1980s. Social media is now in the fabric of our lives, but public controversy still raises questions about the cost-benefit trade-off. Technology and social norms evolve together, yet struggle to synchronize.

Following that first conference, I have built and led CSCW-oriented research labs at Lotus Development Corporation and IBM, bringing a socially engaging group perspective to business products. Much of the work of my group focused on demonstrating the business value of what might otherwise have been seen as frivolous technologies. We saw instant messaging improve productivity and a Facebooklike internal networking system change relationships and flatten hierarchies. Social software applications both inside and outside the workplace are now accepted parts of the infrastructure, critical to effective work and working relationships.

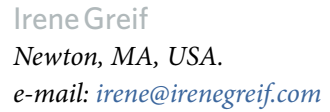

